\title{
ON THE DISTRIBUTION OF SOLUTIONS TO LINEAR EQUATIONS
}

\author{
IGOR E. SHPARLINSKI
}

Macquarie University, Australia

\begin{abstract}
Given two relatively prime positive integers $m<n$ we consider the smallest positive solution $\left(x_{0}, y_{0}\right)$ to the equation $m x-n y=1$. E. I. Dinaburg and Y. G. Sinai have used continued fractions to show that the ratios $x_{0} / n$ are uniformly distributed in $[0,1]$, when $n$ and $m$ run through consequtive integers of intervals of comparable sizes. We use a bound of exponential sums due to W. Duke, J. B. Friedlander and H. Iwaniec to show a similar result when $m$ and $n$ run through arbitrary sets which are not too thin.
\end{abstract}

\section{INTRODUCTION}

Given two positive integers $n$ and $m$ with $\operatorname{gcd}(m, n)=1$, we consider the smallest positive solution $\left(x_{0}, y_{0}\right)$ to the equation $m x-n y=1$. In particular $1 \leq x_{0}<n$, thus the ratios

$$
\rho(m, n)=\frac{x_{0}}{n}
$$

belong to the unit interval $[0,1]$.

E. I. Dinaburg and Y. G. Sinai [2] have used continued fractions to show that the ratios $\rho(m, n)$ are uniformly distributed in [0,1], when $m$ and $n$ run through intervals of the form $\mu_{1} X \leq m \leq \mu_{2} X$ and $\nu_{1} X \leq n \leq \nu_{2} X$ with some fixed $0<\mu_{1}<\mu_{2}<\nu_{1}<\nu_{2}<1$. This result has been improved and generalised by D. Dolgopyat [3], using the continued fraction technique as in [2], and by A. Fujii [5] and G. J. Rieger [8], using the Weil bound on Kloosterman sums, see [6, Corollary 11.12]. We remark that this approach makes no use of the fact that we have two independent variables $m$ and $n$ and

2000 Mathematics Subject Classification. 11D04, 11K38, 11L40.

Key words and phrases. Linear equations, uniform distribution, exponential sums. 
essentially splits the original problem into a sequence of subproblems for each $n \in\left[\nu_{1} X \leq n \leq \nu_{2} X\right]$

Here we show that taking advantage of the bi-variate nature of this problem, one can obtain a more general result on the uniformity of distribution of $\rho(m, n)$ when $n$ and $m$ run through arbitrary sets of integers $\mathcal{M}$ and $\mathcal{N}$, respectively, which are not too thin. As in $[5,8]$ our proof also uses bounds of exponential sums, however instead of the Weil bound we use some bounds due to W. Duke, J. B. Friedlander and H. Iwaniec [4].

Throughout this paper, the implied constants in the symbols ' $O$ ' and ' $\ll$ ' are absolute (we recall that $A \ll B$ and $B \gg A$ are equivalent to $A=O(B)$ ).

\section{Discrepancy and Exponential Sums}

For a sequence of $N$ real numbers $\Gamma=\left(\gamma_{n}\right)_{n=1}^{N}$ of the half-open interval $[0,1)$, denote by $\Delta_{\Gamma}$ its discrepancy, that is,

$$
\Delta_{\Gamma}=\sup _{0 \leq \alpha \leq 1}\left|T_{\Gamma}(\alpha)-\alpha N\right|,
$$

where $T_{\Gamma}(\alpha)$ is the number of points of the sequence $\Gamma$ in the interval $[0, \alpha]$.

We now recall the Erdős-Turán inequality (see [1, 7]), which links the discrepancy with exponential sums.

LEMma 2.1. For any integer $H \geq 1$, the discrepancy $\Delta_{\Gamma}$ of a sequence $\Gamma=\left(\gamma_{n}\right)_{n=1}^{N}$ of $N$ real numbers $\gamma_{1}, \ldots, \gamma_{N} \in[0,1)$ satisfies the inequality

$$
\Delta_{\Gamma} \ll \frac{N}{H}+\sum_{h=1}^{H} \frac{1}{h}\left|\sum_{n=1}^{N} \exp \left(2 \pi i h \gamma_{n}\right)\right| .
$$

We put

$$
\mathbf{e}_{n}(z)=\exp (2 \pi i z / n)
$$

We always follow the convention that arithmetic operations in the arguments of $\mathbf{e}_{n}$ are performed modulo $n$.

Our second main tool is the special case of the bound on certain bilinear sums which is due to W. Duke, J. B. Friedlander and H. Iwaniec [4, Theorem 2].

Lemma 2.2. For arbitrary sets of integers $\mathcal{M} \subseteq[M, 2 M]$ and $\mathcal{N} \subseteq$ $[N, 2 N]$, and any integer $h \in[1, M N]$, the following bound holds

$$
\sum_{n \in \mathcal{N}} \sum_{\substack{m \in \mathcal{M} \\ \operatorname{gcd}(m, n)=1}} \mathbf{e}_{n}\left(h m^{-1}\right) \leq \sqrt{\# \mathcal{M} \# \mathcal{N}}(M N)^{3 / 8}(M+N)^{11 / 48+o(1)}
$$

as $M N \rightarrow \infty$. 


\section{Main Result}

THEOREM 3.1. Let $\gamma>1$ be fixed. Then for arbitrary sets of integers $\mathcal{M}, \mathcal{N} \subseteq[X, \gamma X]$, the discrepancy $D(\mathcal{M}, \mathcal{N})$ of the sequence $\rho(m, n)_{m \in \mathcal{M}, n \in \mathcal{N}}$ satisfies the bound

$$
D(\mathcal{M}, \mathcal{N}) \leq \sqrt{\# \mathcal{M} \# \mathcal{N}} X^{47 / 48+o(1)}
$$

Proof. We cover the interval $[X, \gamma X]$ by $J+1$ halfopen intervals $\left[2^{j} X, 2^{j+1} X\right), j=0, \ldots, J$, where

$$
J=\left\lfloor\frac{\log \gamma}{\log 2}\right\rfloor
$$

whose union covers $[X, \gamma X]$.

Considering subsets of $\mathcal{M}$ and $\mathcal{N}$ from these intervals we see that it is now sufficient to establish the bound in the case $\mathcal{M} \subseteq[M, 2 M]$ and $\mathcal{N} \subseteq[N, 2 N]$ for some $M$ and $N$ with

$$
X \leq M \ll N \ll X
$$

Now, since the function $\mathbf{e}_{n}(z)$ is periodic with period $n$, we derive that for any integer $h$ we have

$$
\exp (2 \pi i h \rho(m, n))=\mathbf{e}_{n}\left(h x_{0}\right)=\mathbf{e}_{n}\left(h m^{-1}\right) .
$$

Therefore, by Lemma 2.2, for any integer $h \in\left[1, X^{2}\right]$, under the condition (3.1), we have

$$
\begin{aligned}
\sum_{n \in \mathcal{N}} \sum_{\substack{m \in \mathcal{M} \\
\operatorname{gcd}(m, n)=1}} \exp (2 \pi i h \rho(m, n)) & =\sum_{n \in \mathcal{N}} \sum_{\begin{array}{r}
m \in \mathcal{M} \\
\operatorname{gcd}(m, n)
\end{array}=1} \mathbf{e}_{n}\left(h m^{-1}\right) \\
& \leq \sqrt{\# \mathcal{M} \# \mathcal{N}} X^{47 / 48+o(1)}
\end{aligned}
$$

Using the estimate (3.2) in a combination with Lemma 2.1, where we choose $H=\left\lfloor X^{2}\right\rfloor$, we conclude the proof.

In particular, we see that for any fixed $\varepsilon>0$, Theorem 3.1 is nontrivial if $\# \mathcal{M} \# \mathcal{N} \geq X^{2-1 / 24+\varepsilon}$.

\section{Comments}

Certainly, using the full power of the results of [4] one can obtain variants of Theorem 3.1 for much more general sets (for example belonging to intervals whose lengths are of different order of magnitude).

We remark that M. S. Risager and Z. Rudnick [9] have recently considered a modification of the original question for the ratios

$$
\vartheta(m, n)=\frac{\sqrt{u_{0}^{2}+v_{0}^{2}}}{\sqrt{m^{2}+n^{2}}},
$$


where $\left(u_{0}, v_{0}\right)$ is the smallest solution to the equation

$$
|m u-n v|=1
$$

and $m$ and $n$ run through the vectors $(m, n)$ of some fixed two-dimensional lattice. Obtaining similar results for $m$ and $n$ from arbitrary sets would be of ultimate interest. Since $\left|u_{0}\right| \leq n$, we derive

$$
\begin{aligned}
\vartheta(m, n) & =\frac{\sqrt{u_{0}^{2}+\left(m u_{0} / n+O(1 / n)\right)^{2}}}{\sqrt{m^{2}+n^{2}}}=\frac{\sqrt{n^{2} u_{0}^{2}+\left(m u_{0}+O(1)\right)^{2}}}{n \sqrt{m^{2}+n^{2}}} \\
& =\frac{\sqrt{n^{2} u_{0}^{2}+m^{2} u_{0}^{2}+O(m n)}}{n \sqrt{m^{2}+n^{2}}}=\frac{u_{0}}{n}\left(1+O\left(1 / u_{0}^{2}\right)\right),
\end{aligned}
$$

the method of this paper can be applied to $\vartheta(m, n)$ as well.

ACKNOWLEDGEMENTS.

The author was supported by ARC grant DP0556431.

\section{REFERENCES}

[1] M. Drmota and R.F. Tichy, Sequences, discrepancies and applications, SpringerVerlag, Berlin, 1997.

[2] E. I. Dinaburg and Y. G. Sinai, The statistics of the solutions of the integer equation $a x-b y= \pm 1$, Funct. Anal. Appl. 24 (1990), 165-171.

[3] D. Dolgopyat, On the distribution of the minimal solution of a linear Diophantine equation with random coefficients, Funct. Anal. Appl. 28 (1994), 168-177.

[4] W. Duke, J. B. Friedlander and H. Iwaniec, Bilinear forms with Kloosterman fractions, Invent. Math. 128 (1997), 23-43.

[5] A. Fujii, On a problem of Dinaburg and Sinai, Proc. Japan Acad. Ser. A Math. Sci. 68 (1992), 198-203.

[6] H. Iwaniec and E. Kowalski, Analytic number theory, American Mathematical Society, Providence, 2004.

[7] L. Kuipers and H. Niederreiter, Uniform distribution of sequences, Wiley-Interscience, New York-London-Sydney, 1974.

[8] G. J. Rieger, Über die Gleichung $a d-b c=1$ und Gleichverteilung, Math. Nachr. 162 (1993), 139-143.

[9] M. S. Risager and Z. Rudnick, On the statistics of the minimal solution of a linear Diophantine equation and uniform distribution of the real part of orbits in hyperbolic spaces, Contemp. Math., Amer. Math. Soc., to appear.

I. E. Shparlinski

Department of Computing

Macquarie University

Sydney, NSW 2109

Australia

E-mail: igor@ics.mq.edu.au

Received: 7.10.2008.

Revised: 3.11.2008. 